\title{
Adrien Thieullen, collectionneur et préhistorien amateur (1833-1913)
}

Adrian Thieullen, collector and amateur prehistorian (1833-1913)

\section{Matthis Gobert}

\section{OpenEdition}

1 Journals

\section{Édition électronique}

URL: http://journals.openedition.org/abpo/2733

DOI : $10.4000 / a b p o .2733$

ISBN : 978-2-7535-3407-0

ISSN : 2108-6443

Éditeur

Presses universitaires de Rennes

Édition imprimée

Date de publication : 27 mars 2014

Pagination : 115-128

ISBN : 978-2-7535-3405-6

ISSN : 0399-0826

\section{Référence électronique}

Matthis Gobert, « Adrien Thieullen, collectionneur et préhistorien amateur (1833-1913) », Annales de Bretagne et des Pays de l'Ouest [En ligne], 121-1 | 2014, mis en ligne le 27 mars 2016, consulté le 01 mai 2019. URL : http://journals.openedition.org/abpo/2733 ; DOI : 10.4000/abpo.2733 


\title{
Adrien Thieullen, collectionneur et préhistorien amateur (1833-1913)
}

\author{
Matthis GOBERT \\ Étudiant, université de La Rochelle
}

Adrien Léopold Thieullen est né à Rochefort le 17 novembre $1833^{1}$. Il quitte assez rapidement la ville pour Paris où il fait ses études au collège Bourbon $^{2}$, puis travaille pendant un temps dans le commerce ${ }^{3}$. Il y réside jusqu'à sa mort en 1913. À partir des années 1870, au plus tard, il s'intéresse à la préhistoire et commence à rassembler un certain nombre d'outils préhistoriques ${ }^{4}$ qui font de lui un collectionneur singulier. Une partie des objets qu'il récolte ne sont en effet pas de véritables outils mais de simples jeux de la nature, des pierres dans lesquelles on peut apercevoir avec plus ou moins de précision des formes humaines ou animales. Une minorité seulement des scientifiques et amateurs d'alors les considèrent comme un témoignage laissé par les hommes préhistoriques. Ces objets sont à l'époque assez communément appelés " pierres figures ${ }^{5}$ ". Ne niant pas qu'il existe des pierres pouvant figurer naturellement de telles formes, Adrien Thieullen affirme cependant qu'il existe des pierres figures à retouches intentionnelles, façonnées par les hommes préhistoriques ${ }^{6}$. En 1907, il fait don de sa collection, qui comprend en partie ces fameuses pierres, au musée de sa ville natale ${ }^{7}$.

1. Arch. dép. de Charente-Maritime, 2E 311/241 (Archives de l'état civil à Rochefort). Registre des naissances, 1833. Acte de naissance de Léopold Adrien Thieullen (18 novembre 1833).

2. L'actuel lycée Condorcet, qui a fréquemment changé de nom au cours du XIX ${ }^{\mathrm{e}}$ siècle.

3. Meunier, Stanislas, "Notice biographique sur A. Thieullen ", Bulletin de la Société d'histoire naturelle d'Autun, vingt-septième bulletin, 1914, p. 2.

4. Le terme d'outil fait référence à des " objets confectionnés et/ou utilisés par les hommes préhistoriques et dont l'analyse conduit à mettre en évidence des activités techniques systématiques et organisées". Voir VIALOU, Denis (dir.), La préhistoire, histoire et dictionnaire, Paris, Robert Laffont, 2004, p. 1020.

5. Bulletin de la Société préhistorique de France, t. III, n 8, 1906, p. 327-329.

6. THIEULLEN, Adrien, " Deuxième étude sur les pierres figures à retouches intentionnelles à l'époque du creusement des vallées quaternaires ", Bulletins et mémoires de la société d'anthropologie de Paris, t. II, 1901, p. 167.

7. Arch. mun. de Rochefort, 3R 500 (Musée, bibliothèque et Beaux-Arts, 1900-1965), 1907. Lettre d'Adrien Thieullen au maire de Rochefort, 20 juillet 1907. 
Thieullen reconnaît aisément l'antiquité géologique de l'homme, admise par les savants notoires dans les années $1860^{8}$. Avec ce nouveau champ de recherches se développent des revues spécialisées, des salles spécifiques sont créées dans les musées et des congrès internationaux sont organisés. Les amateurs, dont fait partie Thieullen, sont réunis dans les sociétés savantes mais peuvent malgré tout être amenés à côtoyer des savants renommés. C'est le cas de Thieullen, qui est cependant assez vite marginalisé à cause de ses théories qui s'écartent du discours dominant ${ }^{9}$.

Le personnage a été relativement peu étudié et on trouve généralement des erreurs dans les courtes notices ou développements qui lui sont consacrés. On peut lire çà et là qu'il est né en $1923^{10}$, qu'il se prénomme André ${ }^{11}$, ou encore qu'il a été "membre d'honneur de la Société d'Histoire naturelle d'Autun, professeur-administrateur du Museum National d'Histoire naturelle [et] président de la Société Géologique de France ${ }^{12}$ ". Il n'en est rien.

S'intéresser à Adrien Thieullen, le collectionneur, c'est évidemment s'intéresser à sa collection. En se référant à la définition de Krzysztof Pomian ${ }^{13}$, il est alors difficile de ne pas s'intéresser conjointement au musée qui devient, au XIX ${ }^{\mathrm{e}}$ siècle, le lieu privilégié de l'exposition de la collection. Enfin, s'intéresser au musée, c'est bien souvent étudier une ou plusieurs donations ${ }^{14}$. Musée, collection, donation et collectionneur sont donc intimement liés et il semble préférable de ne pas traiter de l'un de ces thèmes indépendamment des autres.

8. Richard, Nathalie, "Préhistoire ", dans Delacroix, Christian, DosSE, François, Garcia, Patrick et OfFEnSTADT, Nicolas (dir.), Historiographies, concepts et débats, I, Paris, Gallimard, 2010, p. 578.

9. RICHARD, Nathalie, "Entre matérialisme et spiritualisme : les préhistoriens et la culture dans la seconde moitié du XIX ${ }^{\mathrm{e}}$ siècle ", Ducros Albert, Ducros Jacqueline et Joulian, Frédéric (dir.), La culture est-elle naturelle? Histoire, épistémologie et applications récentes du concept de culture, Paris, Errance, 1998, p. 26.

10. Allary, Michel, Mémoires des rues de Rochefort, Paris, Le Croît Vif, 2006, p. 399-400.

11. Coye, Noël, La Préhistoire en parole et en acte. Méthodes et enjeux de la pratique archéologique (1830-1950), Paris, L'Harmattan, 1997, p. 95-96.

12. BAult, Marie-Pascale, "Adrien Thieullen ", dans Bourdu, Daniel (dir.), Dialogues... Il était une fois la Rue de la Vau Saint-Jacques... et l'Égypte en Poitou-Charentes, Parthenay, Parthenay-Remparts, 1991, p. 31-33.

13. Une collection est «tout ensemble d'objets naturels ou artificiels, maintenus temporairement ou définitivement hors du circuit d'activités économiques, soumis à une protection spéciale dans un lieu clos aménagé à cet effet, et exposés au regard. " Voir Pomian, Krzysztof, Collectionneurs, amateurs et curieux. Paris, Venise XVI -XVIII siècle, Paris, Gallimard, 1987, p. 18.

14. Pomian, Krzysztof, "Musées français, musées européens », dans Georgel, Chantal (dir.), La Jeunesse des musées. Les musées de France au XIX , Paris, RMN, 1994, p. 352. 


\section{Une collection presque comme les autres}

\section{Du biface à la statuette égyptienne}

La collection léguée par Adrien Thieullen est essentiellement préhistorique. Près de cent outils authentiques côtoient une vingtaine de pierres figures. En majorité, ce sont des objets lithiques taillés qui proviennent de différents sites du Bassin parisien : bifaces acheuléens, racloirs, lames du néolithique, burins ou encore haches. Le nombre relativement faible d'objets s'explique par la dispersion d'une partie de la collection au cours de la vie de Thieullen ${ }^{15}$. Le second lot le plus important est composé d'une cinquantaine d'objets antiques d'origine égyptienne. Parmi ceux-ci se trouvent notamment une statuette stéléphore en calcaire datant de la fin de la $\mathrm{XVIII}^{\mathrm{e}}$ dynastie ${ }^{16}$, une statuette de scribe en pierre noire de la XVII ${ }^{\mathrm{e}}$ dynastie et deux fragments en calcaire polychrome, probablement issus de la tombe du pharaon Séthi I ${ }^{\mathrm{er}}$. À ces deux ensembles s'ajoute un mélange hétéroclite de quelques objets médiévaux et asiatiques et de diverses pièces d'artistes contemporains. La collection comprend notamment deux huiles sur toile de Léon Germain Pelouse, deux dessins humoristiques d'Albert Guillaume, un globe terrestre et une sphère armillaire du célèbre fabricant Félix Delamarche. Notons que les fragments de poteries ou de verreries de l'époque médiévale dont fait don Thieullen ont été collectés lors de fouilles rue de l'Abbaye à Paris, alors qu'il cherchait des silex taillés ${ }^{17}$. Cela nuance fortement l'intérêt pour l'archéologie médiévale que l'on pourrait lui attribuer au vu de la présence de ces éléments dans sa collection. Enfin, Thieullen donne sa bibliothèque personnelle, dont l'inventaire ${ }^{18} \mathrm{a}$ été réalisé en 1907 par Léon Ardouin, conservateur de la bibliothèque de la ville de Rochefort. Ce dernier a classé en quatre catégories les près de mille six cents volumes légués par Thieullen : "Anthropologie, préhistoire, archéologie " (361 vol.), "Littérature, histoire, voyages " (905 vol.), "Paris " (164 vol.), et "Sciences" (129 vol.). La grande majorité des ouvrages a été publiée entre le XIX et le début du Xx ${ }^{\mathrm{e}}$ siècle. Si l'ensemble "Littérature, histoire, voyages " semble être sur-représenté, c'est que son intitulé est vague. Les titres restent cependant assez représentatifs du contenu de la bibliothèque, qui ne fait que confirmer les domaines de prédilection de Thieullen. En écartant la bibliothèque, près de 250 objets font partie de la collection léguée par Adrien Thieullen au musée. Elle est à l'image de celles du XIX ${ }^{\mathrm{e}}$ siècle : diversifiée ${ }^{19}$ et avec un nombre non négligeable d'antiquités. La présence d'outils préhistoriques n'est pas non plus un fait rare. Au même

15. MEUNIER, Stanislas, "Notice biographique... », op. cit., p. 3.

16. DEWACHTER, Michel, "L'Égypte des voyageurs et des collections de la région PoitouCharentes ", dans Bourdu, Daniel (dir.), Dialogues... op. cit., p. 23-25.

17. Thieullen, Adrien, "Poteries funéraires, ossements, crânes, etc. de l'époque Mérovingienne ", Bulletins de la Société d'anthropologie de Paris, t. VI, 1895, p. 328-330.

18. Le document est actuellement conservé à la médiathèque de Rochefort.

19. HAmON, Françoise, "Collections : ce que disent les dictionnaires", Romantisme, $\mathrm{n}^{\mathrm{o}} 112,2001$, p. $55-70$. 
titre que les antiquités, ils se collectionnent, s'échangent et s'achètent ${ }^{20}$. L'existence de faussaires témoigne même de l'importance de ce marché. La norme en terme de collection étant l'éclectisme ${ }^{21}$, celle-ci n'a a priori rien d'étonnant. Les pierres figures constituent cependant un élément original, bien qu'Adrien Thieullen ne soit pas le seul à recueillir et à collectionner de tels objets ${ }^{22}$.

\section{Discours et réalité}

Il n'est pas anodin que les discours du collectionneur sur sa collection portent sur ce qui en fait la singularité. Si les pierres figures et autres silex taillés sont conservés et exposés par Thieullen, ils ont avant tout vocation à être analysés. Ces objets archéologiques revêtent sans aucun doute une importance particulière pour lui. En 1905, Adrien Thieullen annonce avoir consacré plus de vingt ans ${ }^{23}$ à ses recherches sur ce qu'il considère être des instruments lithologiques des hommes préhistoriques, dénigrés par la plupart des préhistoriens. Il collectionne ces pierres, qui servent de base à un discours visant à lutter contre ceux qu'il appelle les faux prophètes de la préhistoire, et à exposer aux yeux de tous ce qu'il considère comme la vérité ${ }^{24}$.

Le reste de sa collection est sans doute un subtil et complexe mélange entre passion, prestige et placement ${ }^{25}$ et il semble difficile de déterminer la part qu'il faut attribuer à chacune de ces composantes. Deux lettres de Thieullen adressées au maire de Rochefort nous donnent des éléments de réponse quant à la façon dont il considère sa collection dans son ensemble. Dans une lettre datée du 20 juillet $1907^{26}$, il évoque les « quelques objets et livres " dont il a fait la donation. Près d'un an plus tard, le 6 juillet $1908^{27}$, il parle d'" une collection bien simple qui n'a pris de valeur que par les soins qu'a mis à la cataloguer, à la débrouiller, à la classer M. le docteur Ardouin. " Le crédit à accorder à de tels propos est cependant relativement faible. Ils mettent en avant la modestie, réelle ou de façade, du collectionneur mais n'apportent pas d'éléments déterminants pour cerner davantage le rap-

20. Coye, Noël, La Préhistoire en parole..., op. cit., p. 126.

21. Long, Véronique, "Collections et intérieurs à Paris de 1850 à 1914 ", Hypothèses, 2003, p. 23.

22. Coye, Noël, La Préhistoire en parole..., op. cit., p. 95-96.

23. ThiEUlLEn, Adrien, "Éolithes et autres silex taillés ", Bulletins et mémoires de la Société d'anthropologie de Paris, t. VI, 1905, p. 121.

24. Ibid., p. 112-113.

25. VERBANCK-PIERARD, Annie, "Science et collection. Histoire d'une amitié. La collection d'antiquités classiques de Raoul Warocqué au Musée Royal de Mariemont (Belgique), 1870-1917 ", dans Pomian, Krzysztof, Laurens Annie-France, L'anticomanie. La collection d'antiquités aux XVIII et XIX siècle, Paris, EHESS, 1992, p. 175.

26. Arch. mun. de Rochefort, 3R 500, 1907. Lettre d'Adrien Thieullen au maire de Rochefort, 20 juillet 1907.

27. Arch. mun. de Rochefort, 3R 500, 1908. Lettre d'Adrien Thieullen au maire de Rochefort, 6 juillet 1908. 
port qu'il entretient avec sa collection. Deux traits principaux se dégagent malgré tout du portait d'Adrien Thieullen : sa passion pour la préhistoire et, dans une moindre mesure, son égyptomanie.

\section{Adrien Thieullen : un homme du siècle}

\section{Le préhistorien et ses idées}

À partir de $1879^{28}$, Thieullen devient un fidèle du Muséum d'Histoire naturelle. En 1886, un fait semble avoir été le point de départ de son activité scientifique ${ }^{29}$ : la découverte d'une sépulture préhistorique à Crécy-enBrie $^{30}$, dans la vallée du Grand Morin. L'année suivante, il rejoint la Société d'anthropologie de Paris ${ }^{31}$, devant laquelle il va présenter ses travaux sur la préhistoire. Il n'y a rien d'étonnant à ce qu'ils le soient dans une structure traitant de l'anthropologie, car dans la seconde moitié du XIX siècle, l'anthropologie comprend aussi bien l'ethnographie que l'archéologie préhistorique $^{32}$. Sa première communication date du 21 juillet 1887 . Il y expose ses découvertes préhistoriques ${ }^{33}$ qui ont en commun de témoigner du travail humain. Selon lui, ces pierres qu'il a découvertes mettent à la vue de tous " les vrais instruments de cette civilisation " ${ }^{34}$ ", qui sont alors considérés comme des pierres aux cassures naturelles. Gabriel de Mortillet, un des pionniers qui ont lutté " pour la reconnaissance de la science préhistorique ${ }^{35}$ " lui répond que " surexcité par ces brillants succès [notamment la découverte de la sépulture] ", Thieullen va " un peu trop loin aujourd'hui ${ }^{36}$ ".

Toute l'œuvre de Thieullen peut être présentée comme une lutte pour réhabiliter ces pierres taillées qui auraient échappé à l'attention des anthropologues ${ }^{37}$. En synthétisant, en plus des objets lithiques reconnus par tous, deux types de pierres sont majoritairement récoltés et étudiés par Thieullen : ceux qu'il appelle les " véritables instruments usuels de l'âge de la pierre ", et les pierres figures. Thieullen affirme que sa préoccupation exclusive a été de " réunir les pierres de toute forme, de toute dimension, de toute roche (granit, silex, grès, meulière, calcaire, etc.), sur lesquelles la main de l'homme [préhistorique] avait laissé sa marque; ne rejetant rien a priori, mais collec-

28. Meunier, Stanislas, « Notice biographique... ", op. cit., p. 3.

29. Ibidem, p. 8.

30. Comptes rendus hebdomadaires des séances de l'académie des sciences, t. 103, juilletdécembre 1886, p. 701-702 (séance du lundi 18 octobre 1886).

31. Bulletins de la Société d'anthropologie de Paris, t. X, 1887, p. 6.

32. Dias Nélia, Le musée d'ethnographie du Trocadéro (1878-1908). Anthropologie et muséologie en France, Paris, CNRS, 1991, p. 13.

33. ThiEullen, Adrien " $1^{\circ}$ sur une sépulture... ", Bulletins de la Société d'anthropologie de Paris, t. X, 1887, p. 548-557.

34. Ibidem, p. 553.

35. GRAN-AYMERICH, Ève, Dictionnaire biographique d'archéologie, 1798-1945, Paris, CNRS, 2001, p. 474.

36. Thieullen, Adrien, " $1^{\circ}$ sur une sépulture... ", op. cit., p. 555.

37. Ibidem, p. 550. 
tionnant et classant ces pierres, sans chercher, pour le moment du moins, à en déterminer l'emploi ${ }^{38}$ ". Thieullen ne s'aventura d'ailleurs jamais à livrer une interprétation des productions des hommes préhistoriques. Les "véritables instruments usuels de l'âge de la pierre " ont d'abord été présentés dans un ouvrage publié en $1897^{39}$. Si plusieurs spécialistes ont accepté les idées émises par Thieullen, la plupart les ont accueillies avec hostilité et ont réservé le même traitement au " chercheur dissident ${ }^{40}$ " qu'il était alors. Certains rejettent ces outils parce qu'ils seraient trop nombreux pour avoir été taillés par l'homme préhistorique, d'autres parce qu'ils ne présentent pas les traces de taille habituellement reconnues, ou d'autres encore parce qu'il en existe de trop nombreux types. Stanislas Meunier est élogieux quand il parle de l'homme mais émet, lui aussi, de grandes réserves quand il évoque les théories du chercheur. Ces théories sont donc majoritairement rejetées. Le même traitement est réservé à celles sur les pierres figures, à la différence près qu'il est véritablement raillé pour ces dernières. Là encore, cependant, il n'est pas totalement isolé : d'autres ${ }^{41}$ partagent son avis, mais sans doute pas les savants les plus notables. À Paris, lors du Congrès international d'archéologie et d'anthropologie préhistorique de 1900, il est dénigré par le fameux Sir John Evans, l'un des fondateurs de l'archéologie préhistorique ${ }^{42}$, qui ne manque pas de rappeler l'" imagination trop vive ${ }^{43}$ " dont témoignent les résultats des recherches de Thieullen. Si ses résultats sont parfois moqués, cela n'empêche pas Thieullen de diffuser ses recherches et de rendre visible ce qu'il considère comme la vérité. On notera bien cette persévérance de Thieullen, qui semble ne pas être avare quand il s'agit de faire connaître ses travaux. Bien que ses idées ne soient pas toujours écoutées, il souhaite au moins qu'elles soient lues ou entendues. En plus de ses présentations dans les sociétés savantes, qui sont un espace de liberté où un amateur peut sans encombre présenter le résultat de ses investigations ${ }^{44}$, Thieullen avait des pièces de sa collection exposées dans des grands musées parisiens. Il avait sa vitrine ${ }^{45}$ au musée d'ethnographie du Trocadéro. Au muséum d'histoire naturelle, ses pierres figures et des "silex façonnés, à faciès jusqu'ici inobservés $^{46}$ " étaient exposés dans la galerie de géologie, grâce à Stanislas Meunier,

38. Thieullen, Adrien, "Silex bijoux diluviens », Bulletins et mémoires de la Société d'anthropologie de Paris, t. II, 1901, p. 603.

39. ThiEullen, Adrien, Les véritables instruments usuels de l'âge de la pierre, Paris, Larousse, 1897.

40. Meunier, Stanislas, " Notice biographique... ", op. cit., p. 11.

41. Thieullen, Adrien, "Lettre de M. Thieullen à M. Chauvet ", Bulletins et mémoires de la Société d'anthropologie de Paris, t. IX, 1898, p. 551-553.

42. Gran-AYMERICH, Ėve, Dictionnaire..., op. cit., p. 249.

43. Bulletin de la Société préhistorique de France, t. III, 1906, p. 421.

44. Chaline, Jean-Pierre, Sociabilité et érudition : les sociétés savantes en France XIXe-XXe, Paris, CTHS, 1998, p. 329.

45. ThiEullen, Adrien, "Silex bijoux diluviens ", Bulletins et mémoires de la Société d'anthropologie de Paris, t. II, 1901, p. 352.

46. Thitullen, Adrien, "Le mammouth et le renne à Paris ", Bulletins et mémoires de la Société d'anthropologie de Paris, t. IV, 1903, p. 460. 
un ami de Thieullen, qui était professeur de géologie au Muséum. Thieullen a également eu des pièces exposées dans l'espace réservé à la Société d'anthropologie, lors de l'Exposition Universelle de Paris en 1900.

Thieullen assure qu'il ne connaissait pas les travaux de Boucher de Perthes quand il a commencé à étudier les objets lithiques préhistoriques $^{47}$. Les Antiquités celtiques et antédiluviennes ${ }^{48}$, publiées entre 1849 et 1864, " jettent les fondements de la préhistoire ${ }^{49}$ ". Dans l'ouvrage, cependant, plus de la moitié des planches figurant du matériel archéologique est consacrée " aux cailloux bizarres de toutes sortes ${ }^{50}$ ". Parmi les objets récoltés dans la vallée de la Somme, Boucher de Perthes voit des pierres figures, qu'il interprète comme " une sorte d'alphabet ou de hiéroglyphes préhistoriques. " Après avoir découvert ces idées, Thieullen se présente naturellement comme un continuateur et un fervent admirateur. Il était donc loin d'être le premier à être raillé pour ses pierres figures. Toujours est-il qu'il a apparemment eu un vrai pouvoir de séduction sur un certain nombre de ses contemporains ${ }^{51}$.

Les conclusions de Thieullen se fondent sur plusieurs observations et notamment sur la comparaison entre hommes préhistoriques et " sauvages " actuels. À ce titre, il reprend l'idée, développée notamment par Gabriel de Mortillet ${ }^{52}$ dans les années 1860 , sous l'appellation de " loi de développement similaire de l'humanité ". Selon cette loi, il y aurait analogie entre les civilisations sauvages actuelles et celles des temps préhistoriques. Thieullen déclare donc que si les sauvages taillent aujourd'hui des pierres pour leur donner des formes humaines ou animales, le même phénomène a probablement eu lieu durant la préhistoire ${ }^{53}$. De plus, si les hommes habitant l'abri de la Madeleine ${ }^{54}$ ont pu " graver des figures avec un réalisme extraordinaire $^{55}$ ", des pièces d'art mobilier préhistorique avérées, pourquoi d'autres n'auraient-ils pas pu effectuer de simples retouches sur des pierres préfigurant naturellement un animal ou une partie du corps? Les détracteurs de ces idées répondent, à raison, que les pierres collectées par Thieullen ne présentent pas la trace indubitable du travail humain, et qu'à ce titre, elles doivent être considérées comme résultant de l'action de la nature, et de la nature seulement.

47. Ibidem, p. 472.

48. Boucher De Perthes, Jacques, Antiquités celtiques et antédiluviennes. Mémoire sur l'industrie primitive et les arts à leur origine, 3 vol., Paris, Treutell et Würtz, 1849-1864.

49. GRAN-AYMERICH, Ève, Dictionnaire..., op. cit., p. 102.

50. Cohen, Claudine, Hublin, Jean-Jacques, Boucher de Perthes. Les origines romantiques de la Préhistoire, Paris, Belin, 1989, p. 136

51. Meunier, Stanislas, " Notice biographique... ", op. cit., p. 4-5.

52. Dias, Nélia, Le musée d'ethnographie..., op. cit., p. 108.

53. Thieullen, Adrien, " Deuxième étude sur les pierres figures à retouches intentionnelles à l'époque du creusement des vallées quaternaires ", Bulletins et mémoires de la société d'anthropologie de Paris, t. II, 1901, p. 169.

54. Le gisement du paléolithique qui a donné son nom au magdalénien.

55. ThiEullen, Adrien, "Le mammouth et le renne à Paris ", Bulletins et mémoires de la Société d'anthropologie de Paris, t. IV, 1903, p. 470. 
Stanislas Meunier insinue que, si les assertions de Thieullen ont parfois été si durement rejetées, c'est parce qu'il n'était pas un homme au statut suffisamment honorable ${ }^{56}$. Thieullen est en effet un amateur d'histoire naturelle et de la préhistoire, et reconnaît sans mal ne pas être " un savant de carrière ${ }^{57}$ ". De manière générale, son statut est cependant tout à fait honorable.

\section{Le bourgeois parisien}

Thieullen est membre de plusieurs sociétés savantes : la Société d'anthropologie de Paris, la Société d'excursions scientifiques ${ }^{58}$, ou encore la Société préhistorique de France ${ }^{59}$, dont il est l'un des membres fondateurs. Il correspond au portrait type de l'homme de ces sociétés : " un homme aisé, instruit, d'âge mûr ${ }^{60}$ ". Il a plus de cinquante ans quand il rejoint la Société d'anthropologie de Paris, et plus de soixante-dix quand la Société préhistorique de France est créée. Il est également instruit et aisé. Il a notamment fait rééditer ${ }^{61}$ Les ancêtres d'Adam, histoire de l'homme fossile $^{62}$, initialement paru en 1875, de Victor Meunier, le père de Stanislas. L'ouvrage évoque notamment les travaux de Boucher de Perthes. Cette réédition témoigne de son intérêt pour ce dernier et des questions liées à ses recherches, ce qui n'est plus à démontrer, mais aussi de ses liens avec la famille Meunier et de ses moyens financiers. On peut sans mal affirmer que Thieullen est un bourgeois ${ }^{63}$. Il a de l'argent, du temps ${ }^{64}$, et semble avoir consacré beaucoup des deux à ses recherches archéologiques à partir des années 1880. En 1901, il affirme avoir conduit pendant plus de quinze ans ses recherches seul ${ }^{65}$. Il regrette d'ailleurs de ne pas avoir pu appliquer "la méthode Boucher de Perthes ", qui consistait notamment à former des ouvriers. Travailler avec un nombre réduit de collaborateurs a au moins pu limiter l'exposition de Thieullen aux faussaires, contrairement à Boucher de Perthes qui a volontiers été trompé ${ }^{66}$. Autour de l'année 1900, Adrien Thieullen correspond au prototype de l'oisif. Pas l'oisif véritable, celui qui n'a jamais connu le monde

56. MeuniER, Stanislas, « Notice biographique... », op. cit., p. 33.

57. THiEUllen, Adrien, op. cit., p. 461.

58. Bulletin de la Société d'excursions scientifiques, t. I, 1901, p. 10.

59. Bulletin de la Société préhistorique de France, t. I, no 1, 1904, p. 7.

60. CHALINE, Jean-Pierre, Sociabilité et érudition..., op. cit., p. 223.

61. MeUnIER, Stanislas, " Notice biographique... ", op. cit., p. 41-42.

62. MEunIER, Victor, Les ancêtres d'Adam, histoire de l'homme fossile, Paris, J. Rotschild, 1900.

63. Chaline, Jean-Pierre, Sociabilité et érudition..., op. cit., p. 221.

64. Chaline, Jean-Pierre, "Loisirs et élites sociales : un exemple normand ", dans Daumard, Adeline (dir.), Oisiveté et loisirs dans les sociétés occidentales au XIXe siècle, Abbeville, F. Paillart, 1983, p. 186.

65. Thieullen, Adrien, "Os travaillés à l'époque de Chelles ", Bulletins et mémoires de la Société d'anthropologie de Paris, t. II, 1901, p. 353.

66. Pautrat, Jean-Yves, " Boucher de Perthes : l'invention de l'homme antédiluvien, ou comment devenir un auteur ", dans CARROY, Jacqueline et RICHARD, Nathalie (dir.), 
professionnel, mais l'oisif retiré du monde des affaires. Plus précisément, l'oisif qui est un amateur, occupant ses loisirs dans des secteurs hors de toute préoccupation professionnelle, et notamment dans les recherches érudites et scientifiques ${ }^{67}$.

Comme un certain nombre de bourgeois, et plus particulièrement comme les bourgeois parisiens, Thieullen collectionne. Si, au début du XIX siècle, la passion pour les objets anciens reste le privilège de l'aristocratie ou de marginaux, au cours du siècle elle s'étend à la bourgeoisie et à toutes ses strates. Progressivement, le collectionneur n'est plus vu comme un être fantasque et plus rien ne semble échapper à cette manie de la collection. L'exotisme des objets recherchés est autant géographique qu'historique. Ce n'est cependant pas la collecte d'objets préhistoriques à même le sol qui caractérise le bourgeois, mais l'achat d'objets anciens ${ }^{68}$. Puisque plus ou moins tout le monde collectionne ${ }^{69}$, il ne suffit plus de collectionner pour faire partie de l'élite sociale et culturelle. Les objets de la collection jouent alors un rôle essentiel sur la façon dont est perçu le collectionneur. L'anticomanie de Thieullen ne suffit pas à faire de lui un amateur distingué ${ }^{70}$ dans ce Paris du XIX ${ }^{\mathrm{e}}$ siècle, gorgé " d'objets de toutes les époques et de toutes les provenances ${ }^{71}$ ".

\section{La transmission de la collection}

\section{Donner pour continuer à exister}

En 1907, Adrien Thieullen décide de donner sa collection à la ville de Rochefort. Il semble, de façon générale, être un habitué du " geste philanthropique $^{72}$ ", avec toute l'ambivalence associée au don. En effet, si Thieullen donne sa collection, c'est avant tout pour que ses objets préhistoriques ne disparaissent pas avec lui. Il ne faut pas oublier qu'un don est rarement, si ce n'est jamais, désintéressé, et qu'il faut bien souvent accorder plus d'intérêt à l'intention qu'à la chose donnée ${ }^{73}$. Cela est d'autant plus vrai qu'il est " de la nature du don d'obliger à terme ${ }^{74}$ ". Autrement dit, le donateur

La découverte et ses récits en sciences humaines. Champollion, Freud et les autres, Paris, L'Harmattan, 1998, p. 188.

67. DAUMARD, Adeline, "L'oisiveté aristocratique et bourgeoise en France au XIX ${ }^{\mathrm{e}}$ siècle : privilège ou malédiction?", dans DAUMARD, Adeline (dir.), Oisiveté et loisirs..., op. cit., p. 130.

68. CHARPY, Manuel, " L'ordre des choses. Sur quelques traits de la culture matérielle bourgeoise parisienne, 1830-1914", Revue d'histoire du XIX siècle, n 34, 2007, p. 105.

69. Maupassant, Guy de, "Bibelots ", Le Gaulois, 22 mars 1883.

70. PETY, Dominique, «Le personnage du collectionneur au XIX ${ }^{\mathrm{e}}$ siècle : de l'excentrique à l'amateur distingué ", Romantisme, $\mathrm{n}^{\circ} 112,2001, \mathrm{p} .71-81$.

71. ChARPY, Manuel, "L'ordre des choses... ", op. cit., p. 105.

72. Poulot, Dominique, "Musées et collections : pour une histoire de la patrimonialité ", Histoire de l'art, $\mathrm{n}^{\circ} 62$, avril 2008, p. 7 .

73. SÉNÈQUE, Les bienfaits, Livre I, VI, Paris, Gallimard, 1996, p. 68.

74. Mauss, Marcel, Essai sur le don, Paris, Presses Universitaires de France, 2007, p. 138. 
contraint le donataire à rendre, d'une façon ou d'une autre, ce qui a été donné.

Parmi les dons ou autres libéralités que Thieullen a pu faire, l'érection d'une statue en $1908^{75}$, en l'honneur de Boucher de Perthes, est particulièrement révélatrice du phénomène. Ériger un monument à sa gloire, c'est reconnaître le statut particulier du personnage. Or ce statut est lié aux idées qu'il a développées et défendues, et qui se trouvent, en partie, être celles que partage Thieullen. En 1902 déjà, il revendiquait un "monument international élevé à l'homme de génie ${ }^{76}$ " qu'était Boucher de Perthes. Reconnaître le génie de Boucher de Perthes, pour Thieullen, c'est reconnaître la validité de toutes ses théories. Si certains ne manquent pas de lui faire remarquer que les thèses de Boucher de Perthes sont admises par les préhistoriens, Thieullen milite cependant pour que toutes le soient, y compris celles sur les pierres figures. Il est particulièrement intéressant de remarquer que le statuaire à l'origine du monument, Emmanuel Fontaine, a également réalisé un buste d'Adrien Thieullen ${ }^{77}$ après sa mort. Le phénomène n'est pas si rare ${ }^{78}$, mais il est loin d'être anodin. Cela renforce davantage la panthéonisation du collectionneur qui trouve alors " dans les salles qui lui sont dédiées une forme de mausolée ${ }^{79}$ ".

Si toute collection témoigne du goût et du savoir du collectionneur ${ }^{80}$, il semble que toute donation témoigne d'un désir de reconnaissance. Pour Thieullen, cela va plus loin que la reconnaissance de son goût; il veut mettre sa pensée en lieu sûr ${ }^{81}$. Plus encore que sa collection et ses talents de collectionneur, il souhaite que ses idées soient reconnues. Exposer le support de ses idées au musée, c'est alors, sans nécessairement affirmer la validité des théories, reconnaître leur intérêt. On retrouve là le projet qui préside à la donation de certaines collections : l'éducation des visiteurs ${ }^{82}$. Le plus souvent, cette éducation est esthétique, ici elle est scientifique. Il s'agit d'exposer aux yeux de tous ce qui serait à tort dénigré par les scientifiques, et de faire ainsi éclater la vérité. La donation devient alors un acte d'utilité publique. La présence de pièces de la collection d'Adrien Thieullen au musée n'est cependant pas, avec la donation à Rochefort, un

75. Bulletins et mémoires de la Société d'anthropologie de Paris, t. IX, 1908, p. 539-542.

76. ThiEullen, Adrien, "Le préchelléen en Belgique ", Bulletins et mémoires de la Société d'anthropologie de Paris, t. III, 1902, p. 767.

77. Arch. municipales de Rochefort, 3R 500 : 1914. Lettre de la sœur d'Adrien Thieullen au maire de Rochefort, 9 juillet 1914 .

78. Agulhon, Maurice, " La statue de grand homme. Critique politique et critique esthétique ", Mil neuf cent, $\mathrm{n}^{\circ}$ 21, 2003, p. 9-19.

79. CABAnÈs, Jean-Louis et Pety, Dominique, "Avant-propos ", Romantisme, n 112, 2001, p. 5

80. Pomian, Krzysztof, "Collections : une typologie historique ", Romantisme, $\mathrm{n}^{\circ} 112$, 2001, p. 21.

81. ARDOUIN, Léon, " Dons récents faits au Musée et à la Bibliothèque de la Ville ", Bulletin de la société de géographie de Rochefort, t. XXIX, nº 4, 1907, p. 357.

82. LONG, Véronique, " Les collectionneurs d'œuvres d'art et la donation au musée à la fin du XIX ${ }^{\mathrm{e}}$ siècle : l'exemple du musée du Louvre ", Romantisme, nº 112, 2001, p. 51. 
fait nouveau : il exposait déjà des éléments de sa collection dans les vitrines de musées parisiens. La différence est grande cependant entre quelques pièces d'histoire naturelle dans un musée parisien dont c'est la spécialité, et l'ensemble d'une collection diversifiée dans un musée municipal de province. D'ailleurs, $s$ 'il est possible en province de mélanger collections d'archéologie, d'ethnographie et d'histoire naturelle, ce n'est pas le cas à Paris, où il existe des musées spécialisés, ou tout au moins des salles particulières ${ }^{83}$.

On comprend mieux pourquoi Thieullen se sépare de sa collection. Si cela peut être vu comme un dépouillement, il évite au pire la dispersion et au mieux assure une visibilité supérieure à ses idées. Il exprime d'ailleurs le chagrin qu'il ressent en imaginant que ce qu'il a rassemblé avec tant de peine et tant de plaisir puisse disparaître avec lui ${ }^{84}$.

\section{Une reconnaissance sociale}

La donation de Thieullen à la ville de Rochefort est présentée comme un heureux hasard, mais il n'est pourtant pas rare qu'un collectionneur lègue sa collection à sa ville natale ${ }^{85}$. Il n'est pas rare non plus que soit accordé un nom à la salle où est conservée la collection d'une donation. Auguste Marguillier ${ }^{86}$ dénonce la vanité des collectionneurs-donateurs du Louvre, qui veulent tous avoir leur salle à part, et à leur nom. Le nom de la salle n'est cependant pas toujours uniquement celui du donateur. On a ici un parfait exemple du phénomène, puisque Léon Ardouin est associé avec Thieullen à la reconnaissance de la ville : on nomme le lieu qui accueille la collection " salle Thieullen et Ardouin ${ }^{87}$ ". C'est un témoignage supplémentaire du rôle important qu'il a pu jouer dans la transmission de cette collection. Pour le musée, il s'agit aussi de rendre hommage à ceux qui sont bien souvent natifs de la ville. Que les collections soient locales ou non, en termes de provenance géographique, c'est une forme d'autocélébration ${ }^{88}$ : on met en avant ceux qui sont nés ici, qui ont été les mécènes du lieu, et qui vont y rester plus ou moins longtemps grâce à leur collection. Il s'agit aussi d'exprimer publiquement des remerciements pour encourager d'autres donateurs. Après sa mort en 1913, Thieullen est cité comme " un enfant de Rochefort qui a voulu prendre sa place parmi les bienfaiteurs de la ville ${ }^{89}$ ",

83. Dias, Nélia, Le musée d'ethnographie..., op. cit., p. 105.

84. Ardouin, Léon, " Dons récents... ", op. cit., p. 357.

85. GEORGEL, Chantal, "Le musée, lieu d'enseignement, d'instruction et d'édification ", dans GEORGEL, Chantal (dir.), La Jeunesse des musées. Les musées de France au XIX RMN, 1994, p. 68.

86. Marguillier, Auguste, "Musées et collections ", Mercure de France, $1^{\text {er }}$ juin 1914, p. 639 .

87. Arch. mun. de Rochefort, Délibérations du Conseil municipal : 24 nov. 1906-13 oct. 1909. Séance du $1^{\text {er }}$ juillet 1908.

88. Poulot, Dominique, Une histoire des musées de France, XVII $-X X^{e}$ siècle, Paris, La Découverte, 2008, p. 134.

89. Tablettes des deux Charentes, samedi 12 avril 1913. 
et des proches vont toujours admirer sa collection au musée ${ }^{90}$. Donner au musée, c'est donc bien vouloir que sa collection soit exposée dans ce lieu spécifique, qui devient son sanctuaire. Pour la ville, recevoir la collection est une véritable bonne fortune ${ }^{91}$; cela permet d'accroître facilement les collections du musée sans engager trop de dépenses. C'est un motif de satisfaction lorsque l'on sait qu'il est parfois difficile pour les musées d'enrichir leurs collections par des achats ${ }^{92}$, faute de moyens financiers. Bien que la collection de Thieullen soit réceptionnée par le musée, elle ne peut cependant continuer à exister que si elle reste maintenue hors du circuit économique, est "soumise à une protection spéciale dans un lieu aménagé à cet effet ", et est exposée au regard. Le musée remplit parfaitement ces conditions, le plus souvent en tout cas.

Même s'il est dans l'erreur, Thieullen consacre en grande partie les trente dernières années de sa vie à ses recherches archéologiques. Il ne ménage ni son temps, ni son argent, et encore moins sa réputation. Il est maintes fois raillé pour ses idées mais les défend ardemment, à la suite de celui qu'il n'a pas connu mais qu'il considère comme son maître : Boucher de Perthes. Comme lui, il œuvre pour faire connaître ses découvertes et pour exister en tant que savant ${ }^{93}$. Une partie des objets préhistoriques collectionnés par Thieullen sont en effet de faux outils préhistoriques. Ce ne sont pas des copies du XIX ${ }^{\mathrm{e}}$ siècle, mais des objets anciens considérés pour ce qu'ils ne sont pas. Si la différence est notable, on peut tout de même rapprocher ces deux types d'objets factices et les conséquences qu'il y a sur leur exposition. Ces objets ne sont jamais exposés bien longtemps. Les cailloux étranges de Boucher de Perthes ont de la même manière été rapidement cachés ${ }^{94}$ aux visiteurs. Thieullen précise d'ailleurs qu'il n'a jamais eu l'occasion de les voir lors de ses visites à Abbeville ${ }^{95}$ ou au musée de Saint-Germain. Différentes conceptions du rôle du musée s'opposent ici. Une première conception consacre le rôle d'éducation. C'est en partie ce qui explique pourquoi Adrien Thieullen souhaitait que ses pierres figures soient exposées. Il s'agissait aussi plus simplement, pour lui, de montrer une œuvre d'art qui viendrait de temps très reculés. Deux desseins sont donc derrière cette exposition : un pédagogique et un qui relève davantage de l'esthétique et de la curiosité. D'autres conditions peuvent être ajoutées pour justifier l'exposition d'un objet, et notamment un critère de

90. Arch. mun. de Rochefort, 3R 500 : 1916. Lettre de la sœur d'Adrien Thieullen au maire de Rochefort, $1^{\text {er }}$ décembre 1915.

91. ARDOUIN, Léon, "Dons récents... ", op. cit., p. 357.

92. LAPAUZE, Henry, Les Musées de province, rapport, enquête, législation, Paris, PlonNourrit, 1908, p. 67.

93. PAUTRAT, Jean-Yves, « Boucher de Perthes : l'invention de l'homme antédiluvien, ou comment devenir un auteur", loc. cit, p. 192.

94. Cohen, Claudine, Hublin, Jean-Jacques, Boucher de Perthes..., op. cit., p. 237.

95. ThiEulLEN, Adrien, "Le préchelléen en Belgique ", Bulletins et mémoires de la Société d'anthropologie de Paris, t. III, 1902, p. 762. 
vérité, c'est une autre conception du rôle du musée, qui n'est d'ailleurs pas toujours incompatible avec la première. Les objets exposés doivent être "vrais ". Certains faux sont pourtant loin d'être dénués d'intérêt ${ }^{96}$. Si le musée expose les témoins matériels de l'homme du passé, les pierres figures peuvent alors être concernées. Elles ne témoignent pas de l'homme du paléolithique ou du néolithique, mais de l'homme du XIX siècle qui collectionne les pierres qu'il étudie. C'est ce critère de vérité qui a sans doute conduit à éclipser les pierres figures.

Avec sa donation en 1907, Thieullen participe à l'élaboration du temple dédié à sa collection. Elle est cependant rapidement mutilée : d'abord exposée, elle est ensuite amputée des pierres figures qui en sont la substance ${ }^{97}$. Après avoir symboliquement représenté l'invisible ${ }^{98}$, le lointain temps des hommes préhistoriques, sanctuarisées et intouchables derrière une vitrine, les pierres figures si chères à Adrien Thieullen sont littéralement devenues invisibles. La collection n'existe alors plus vraiment. Une grande partie en étant cachée, elle n'est plus l'expression de la personnalité de Thieullen qui, bien qu'affirmant ne pas être doté de l'esprit inventif de Jules Verne ${ }^{99}$, semblait être doué d'une imagination certaine.

96. Hellmann, Marie-Christine, citée par Ghiron-Bistagne, Paulette, " Un faux exemplaire au musée Borély ", dans Pomian, Krzysztof, Laurens, Annie-France, L'anticomanie..., op. cit., p. 346.

97. Les pierres figures ne sont plus exposées depuis une date comprise entre 1930 et 1948 .

98. Pomian, Krzysztof, Collectionneurs, amateurs et curieux. Paris, Venise XVI ${ }^{e}$-XVIII $I^{e}$ siècle, Paris, Gallimard, 1987, p. 42.

99. Thieullen, Adrien, "Éolithes et autres silex taillés ", Bulletins et mémoires de la Société d'anthropologie de Paris, t. VI, 1905, p. 124. 


\section{RÉSUMÉ}

Adrien Thieullen (1833-1913) est un préhistorien amateur né à Rochefort, qui vit à Paris, où il exerce la majeure partie de ses activités scientifiques. Il expose au sein de plusieurs sociétés savantes des théories controversées sur la préhistoire et notamment sur les pierres figures, des cailloux auxquels la nature a donné des formes humaines ou animales. Allant contre la position des scientifiques de renom de l'époque, Adrien Thieullen reprend en partie la théorie de Jacques Boucher de Perthes et défend l'idée qu'ils ont été taillés par l'homme préhistorique. Outre ces pierres figures et d'authentiques outils préhistoriques, Adrien Thieullen collectionne, comme de nombreux bourgeois parisiens, des objets d'époques et d'horizons divers. Malgré son goût prononcé pour l'Égypte, c'est bien la préhistoire qui passionne Thieullen. Animé par le désir de faire reconnaître ses théories, il ne cesse de discourir sur ses découvertes et donne sa collection au musée de sa ville natale en 1907.

\section{ABSTRACT}

Adrien Thieullen (1833-1913) was an amateur prehistorian. He was born in Rochefort but lived in Paris, where he had most of his scientific activities. A member of several learned societies, he expounded controversial theories about prehistory - most notably about "figured stones", rocks to which nature has given human or animal shapes. Standing against the viewpoint of the renowned specialists of his time, Adrien Thieullen had partly taken up Jacques Boucher de Perthes's theory that these rocks had been carved by Prehistoric man. In addition to these "figured stones" and other genuine prehistoric tools, Adrien Thieullen was a collector of antique objects from all over the world - a passion shared by many Parisian bourgeois of his time. Though he was very interested in ancient Egypt, he was mostly fascinated by prehistory. Eager to see his theories recognised, he relentlessly gave talks about his discoveries. In 1907 he donated his collection to the museum of Rochefort. 